\title{
ROBUST LQR AND LQI CONTROL WITH ACTUATOR FAILURE OF A 2DOF UNMANNED BICYCLE ROBOT STABILIZED BY AN INERTIAL WHEEL
}

\author{
ADAM OWCZARKOWSKI $^{a, *}$, DARIUSZ HORLA ${ }^{a}$ \\ ${ }^{a}$ Institute of Control and Information Engineering \\ Poznań University of Technology, ul. Piotrowo 3a, 60-965 Poznań, Poland \\ e-mail: adam.j.owczarkowski@doctorate.put.poznan.pl, \\ dariusz.horla@put.poznan.pl
}

\begin{abstract}
Essential ingredients for robust control are the ability to cope with different types of system behavior following modeling imperfections and the ability to assure a certain performance level. In this paper, we propose to use an actuator fault-tolerant control law to govern, during experiments, the stabilization of a bicycle robot with an inertial wheel in order to take into account unmodeled uncertainty introduced by using a linearized model in an LQR fashion. Our proposal is illustrated by signal plots and the values of performance indices obtained from a set of experiments.
\end{abstract}

Keywords: unmanned bicycle robot, LQR/LQI control, robustness, actuator failure.

\section{Introduction}

The problem of stabilization, or robust control, is widely discussed in the literature. Some papers are related to performance measures as a quadratic cost for uncertain systems, as the works of Chang and Peng (1972) or Petersen and McFarlane (1992) for the case of continuous-time control systems. In the case of discrete-time control systems, the guaranteed-cost control has been addressed by, e.g., Xie and Soh (1995).

Control algorithms, when implemented, are prone to failures. Thus it is important to use controllers that can tolerate these failures, to guarantee stability and a certain level of performance. This paper adopts the approach presented by Yang et al. (2000b) to apply LQR/LQI control strategies to stabilize a bicycle robot in an unstable equilibrium point, based on its linearized model. The actuator failure feature of the controller is used to introduce the uncertainty that originates from adopting a linearized model to control a nonlinear system that can be modeled by nonlinear state-space equations. Appropriate results are shown on the basis of a set of performed experiments with a bicycle robot.

The state-feedback control law is used to guarantee the cost in the case of actuator failure or, in other words, to assure that the lack of consistency between the linearized

*Corresponding author model and the "true" model of the plant is compensated, projecting the "robustness area" on the static characteristic of the nonlinearity present in the control system or an unknown static characteristic of the nonlinear system.

The principle and basic introduction to inertial wheel stabilization can be found in the work of Owczarkowski et al. (2014). The LQR approach to stabilize an unmanned bicycle is presented by Yang et al. (2011) or Smerpitak et al. (2012), but only as a result of simulations and no robustness introduced to the control system. The dynamic characteristics, i.e., the response of the closed-loop system to the initial conditions presented in this paper, are similar to the ones presented in the above-cited works, but they can take the uncertainty of the linearized model of the robot into account.

\section{Experimental setup: An unmanned bicycle robot}

Figure 1 shows a real robot. It has two degrees of freedom (the deflection angle from the vertical position and the angle of the reaction wheel) and one actuator (electric motor). The main goal of the control system is to stabilize the robot at an unstable equilibrium point using as little energy as possible. The DC brushed electric motor is fitted inside the construction and accelerates or decelerates the rotating mass (the steering wheel, the reaction wheel). 


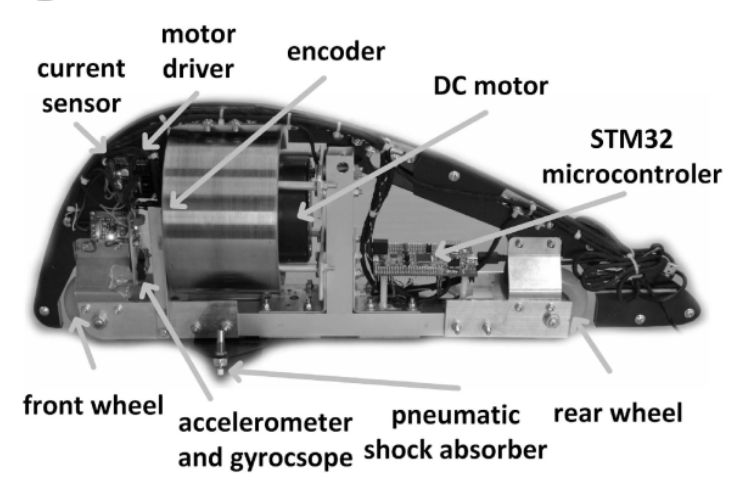

Fig. 1. Picture of a real robot.

The motor shaft torque creates a reaction which is used for stabilization and is the core principle of operation of this robot. The only control signal is the electric motor current proportional to the torque, which makes the direct torque control absolutely vital and fundamental in this case. The measurement system consists of three sensors: a one-axis accelerometer, a one-axis gyroscope and an encoder. Raw measurement values would be useless without a specially prepared filtering and estimation process. To obtain a reliable state vector, we decided to use a combination of Kalman and FIR filters. The studies presented by Drapikowski et al. (2012) prove that this measurement system is sufficient for this kind of robot. Every calculation is made by an embedded system with a high performance 32-bit STM32 microcontroller.

There are some constraints in the system that must be taken into account, namely, the maximum angle from the vertical position, the maximum velocity of the reaction wheel and the maximum control value. In other words, every electric motor has its own limits, i.e., the maximum velocity and the maximum torque. If one of these values saturates, the system becomes much more difficult to control. In such a situation, the actuator is uncontrollable. This article shows how to cope with actuator failures using modified LQR control.

\section{Model of the robot}

3.1. Continuous-time 2DOF model of the plant and the solution procedure for nonlinear difference equations. The full mathematical model of the robot is defined by the following non-linear differential equations (time index omitted for brevity):

$$
\begin{aligned}
\dot{x}_{1}(t)= & x_{2}(t), \\
\dot{x}_{2}(t)= & \frac{g h_{r} m_{r} \sin \left(x_{1}(t)\right)}{I_{r g}}-\frac{b_{r} x_{2}(t)}{I_{r g}} \\
& -\frac{b_{I} x_{4}(t)}{I_{r g}}+\frac{k_{m} u(t)}{I_{r g}},
\end{aligned}
$$

$$
\begin{aligned}
\dot{x}_{3}(t) & =x_{4}(t), \\
\dot{x}_{4}(t) & =\frac{k_{m} u(t)}{I_{I}+I_{m r}}-\frac{b_{I} x_{4}(t)}{I_{I}+I_{m r}},
\end{aligned}
$$

where (see Fig. 2 for the kinematic scheme of the object) $\underline{x}$ is the state vector, $x_{1}$ is the angle of the robot from the vertical, $x_{2}$ is the angular velocity of the robot, $x_{3}$ is the rotation angle of the reaction wheel, $x_{4}$ is the angular velocity of the reaction wheel, $u$ stands for control, $u_{i}$ is the current of the $i$-th motor, $m_{r}$ is the weight of the robot, $I_{I}$ is the moment of inertia of the reaction wheel, $I_{m r 1}$ is the moment of inertia of the rotor of the motor $1, I_{r g}$ is the moment of inertia of the robot relative to the ground, $h_{r}$ is the distance from the ground to the center of mass of the robot, $g$ is the gravity of the Earth, $k_{m i}$ is the $i$-th motor constant, $b_{r}$ is the coefficient of friction in the robot rotation, $b_{I}$ is the coefficient of friction in the rotation of the reaction wheel, $P_{1}, P_{2}$ are contact points of the wheels with the ground, $C_{1}$ is the center of the rear wheel, $C_{2}$ is the center of the front wheel, $C O M$ stands for the center of mass.

The above model takes centrifugal force, gravitation force and also the reaction momentum from an inertial drive into account. It has been derived with reference to the rules described by Block et al. (2007), and yet, apart from taking the listed phenomena into account, it is a simplification of reality to some extent.

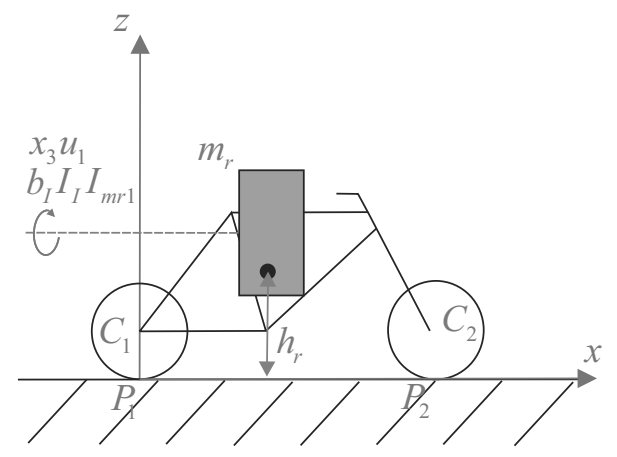

(a)

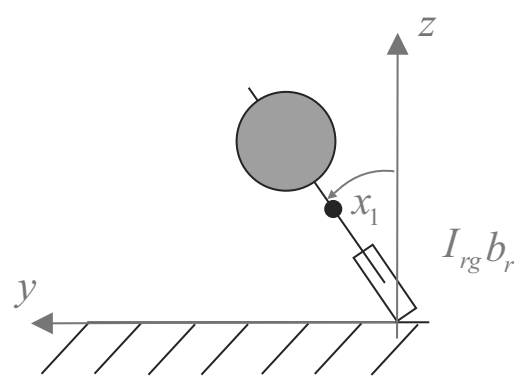

(b)

Fig. 2. Kinematic scheme of a bicycle robot: main view (a), side view (b).

The system can be linearized by a Jacobian matrix to 
the form $\underline{\dot{x}}(t)=\boldsymbol{A}_{l} \underline{x}(t)+\underline{b}_{l} u(t)$, where

$$
\begin{aligned}
\boldsymbol{A}_{l} & =\left[\begin{array}{cccc}
0 & 1 & 0 & 0 \\
\frac{g h_{r} m_{r}}{I_{r g}} & -\frac{b_{r}}{I_{r g}} & 0 & -\frac{b_{I}}{I_{r g}} \\
0 & 0 & 0 & 1 \\
0 & 0 & 0 & -\frac{b_{I}}{I_{I}+I_{m r}}
\end{array}\right], \\
\underline{b}_{l} & =\left[\begin{array}{c}
0 \\
\frac{k_{m}}{I_{r g}} \\
0 \\
\frac{k_{m}}{I_{I}+I_{m r}}
\end{array}\right],
\end{aligned}
$$

with the linealization point (units have been omitted here) $\underline{x}_{l}=[0.5,0,0,0]^{T}$. The physical model of the robot has the following parameters: $m_{r}=$ $3.96 \mathrm{~kg}, h_{r}=0.13 \mathrm{~m}, I_{I}=0.0094 \mathrm{~kg} \cdot \mathrm{m}^{2}, I_{m r 1}=$ $0.0008 \mathrm{~kg} \cdot \mathrm{m}^{2}, I_{r g}=0.0931 \mathrm{~kg} \cdot \mathrm{m}^{2}, r_{w}=0.03 \mathrm{~m}, g=$ $9.80665 \mathrm{~m} \cdot \mathrm{s}^{2}, \lambda=\pi / 2 \mathrm{rad}, k_{m 1}=0.421 \mathrm{~N} \cdot \mathrm{m} \cdot \mathrm{A}^{-1}$, $b_{r}=0.0013 \mathrm{~N} \cdot \mathrm{m} \cdot \mathrm{s}, b_{I}=0.0003 \mathrm{~N} \cdot \mathrm{m} \cdot \mathrm{s}$, identified by means of previous experiments or appropriate measurements.

3.2. Discrete-time model of the plant. In order to apply the controller on hardware, the following multivariable model of the linearized plant must be introduced:

$$
\begin{aligned}
\underline{x}_{t+1} & =\boldsymbol{A} \underline{x}_{t}+\underline{b} u_{t}, \\
\underline{y}_{t} & =\boldsymbol{C} \underline{x}_{t},
\end{aligned}
$$

where the appropriate matrices and vectors have known sizes and are the result of step-invariant discretization of the continuous-time model of the plant (5), 6), with a chosen sample period $T_{S}$. The output vector $\underline{y} \in \mathbb{R}^{p}$, the constrained control signal $u$, and the state vector $\underline{x} \in \mathbb{R}^{n}$ are in the discrete-time domain (denoted henceforth by the subscript $t$ ).

\section{Actuator failure models}

The LQR-type approach to control a bicycle robot assuring some degree of robustness to actuator failures is applied in the paper. In the proposed algorithm, the control performance index is defined as in the standard LQR control (Kwakernaak and Sivan, 1972),

$$
J=\sum_{t=0}^{\infty}\left(\underline{x}_{t}^{T} \boldsymbol{Q} \underline{x}_{t}+R u_{t}^{2}\right),
$$

with the weighing matrix $Q \geq 0$, and $R \geq 0$. In the most general case, one can assume the actuator failure model as in the work of Zuo et al. (2010), i.e.,

$$
u_{t}^{k}=\left(1-\rho_{t}^{k}\right) \operatorname{sat}\left(v_{t} ; \alpha\right), \quad k=1, \ldots, g_{F},
$$

where $\rho_{t}^{k}$ is an unknown constant from the span that will be defined in a further part of the text, index $k$ denotes the $k$-th failure model, and $g_{F}$ is the total number of failure models. The symbol $u_{t}^{k}$ refers to the constrained control signal, assuming that an actuator failure takes place (in the other case, $u_{t}^{k}=v_{t}$ ). For any actuator failure model, including the situation for constraints imposed on the control signal, the constant $\rho_{t}^{k}$ lies in $\rho_{-, t}^{k} \leq \rho_{t}^{k} \leq$ $\rho_{+, t}^{k}$, and the function sat defines the method of applying constraints (e.g., a cut-off constraint).

Having taken a single model of failure into account, (10) can be transformed (Yang et al., 2000b; 2000a; Horla and Królikowski, 2011) to

$$
u_{t}^{F}=\varrho v_{t},
$$

where

$$
0 \leq \varrho_{-} \leq \varrho \leq \varrho_{+},
$$

with $\varrho_{-} \leq 1$ and $\varrho_{+} \geq 1$. This enables us to mimic uncertainty introduced by the linearized model with a kind of actuator failure-the control signal applied to the system causes different behavior, as expected. Furthermore, for the robot considered, current constraints introduce additional failure-like behavior (inability to generate computed, i.e., required, torques).

With reference to 10$], \varrho_{-}=\varrho_{+}$means that there are no active constraints imposed on the control signal or no failures have taken place, and $u_{t}^{F}=v_{t}$. The case $\varrho_{-}>0$ corresponds to a partial failure, and $\varrho_{-}=0$ to the outage case.

In order to streamline the further presentation, the following notation has been adopted from Yang et al. (2000a) as well as Horla and Królikowski (2011):

$$
u_{t}=u_{t}^{F} \text {. }
$$

\section{Control strategies considered}

Firstly, the LQR control law of the form

$$
v_{t}=\underline{k}^{T} \underline{x}_{t}
$$

is considered, where $\underline{x}_{t}$ is the sampled state vector from the robot, measured by means of a suitable combination of filtering and estimation of signals from the accelerometer, the gyroscope and the encoder. It refers to the equations of the nonlinear model of the plant (1)-(4). The latter control law is called reliable and assures that a specified value of the performance index (9) is not exceeded for the given plant model (referring to the real-world control system), if it is connected to a certain matrix $\boldsymbol{P}$, the system (7), (8), and if $\boldsymbol{P}$ satisfies the inequality (Yang et al., 2000a; Horla and Królikowski, 2011)

$$
\begin{aligned}
\left(\boldsymbol{A}+\varrho \underline{b k}^{T}\right)^{T} \boldsymbol{P}\left(\boldsymbol{A}+\varrho \underline{b k^{T}}\right) & \\
& -\boldsymbol{P}+R \varrho^{2} \underline{k k}^{T}+\boldsymbol{Q} \leq 0 .
\end{aligned}
$$


The closed-loop model of the system

$$
\underline{x}_{t+1}=\left(\boldsymbol{A}+\varrho \underline{b k^{T}}\right) \underline{x}_{t}
$$

is then stable, and the performance index over an infinite horizon satisfies

$$
J=\sum_{t=0}^{\infty} \underline{x}_{t}^{T}\left(\boldsymbol{Q}+R \varrho^{2} \underline{k k}^{T}\right) \underline{x}_{t} \leq \underline{x}_{0}^{T} \boldsymbol{P} \underline{x}_{0} .
$$

If no robustness against the actuator failure is taken into consideration, the optimal state-feedback vector $\underline{k}$ for the control law (14) is derived as a solution of the set of equations

$$
\begin{aligned}
\underline{k}^{T}= & -\left(\underline{b}^{T} \boldsymbol{P} \underline{b}+R\right)^{-1} \underline{b}^{T} \boldsymbol{P} \boldsymbol{A}, \\
\boldsymbol{P}= & \boldsymbol{Q} \boldsymbol{A}^{T} \boldsymbol{P} \boldsymbol{A} \\
& -\boldsymbol{A}^{T} \boldsymbol{P} \underline{b}\left(\underline{b}^{T} \boldsymbol{P} \underline{b}+R\right)^{-1} \underline{b}^{T} \boldsymbol{P} \boldsymbol{A},
\end{aligned}
$$

with an optimal value $J_{\underline{k}}$ of the performance index (9), based on deriving $\underline{k}$ according to (18) and (19), being at the same time the upper boundary of (17), given as

$$
J_{\underline{k}}=\underline{x}_{0}^{T} \boldsymbol{P} \underline{x}_{0} .
$$

Secondly, the LQI control law is also taken into account,

$$
v_{t}=\underline{k}^{T} \underline{x}_{t}+\underline{k}_{I}^{T} \underline{x}_{I, t},
$$

where $\underline{x}_{t}$ is the measured state vector of the robot, as in the latter case, and $\underline{x}_{I, t}$ is an appropriate integral of the state vector, whereas $\underline{k}_{I}$ for the described problem is a zero vector with the third element with the value $a \neq 0$ (compensation of the gyroscopic drift in $x_{3}$ ).

\section{Derivation of an optimal state-feedback vector in the case of an actuator failure}

The following algorithm (Yang et al., 2000a) enables derivation of the optimal state-feedback vector $\underline{k}$ to increase the robustness of the system against actuator failure:

1. solve (19) with respect to $\boldsymbol{P}$ (mark the result as $\boldsymbol{P}^{*}$ ), and choose an arbitrary $R_{0}$, satisfying

$$
R_{0} \leq\left(\underline{b}^{T} \boldsymbol{P}^{*} \underline{b}+R\right)^{-1}
$$

2. solve

$$
\boldsymbol{P}=\boldsymbol{Q}+\boldsymbol{A}^{T} \boldsymbol{P} \boldsymbol{A}-\boldsymbol{A}^{T} \boldsymbol{P} J_{0} \boldsymbol{P} \boldsymbol{A}
$$

with respect to the stabilizing $\boldsymbol{P}$ and check the condition

$$
R_{0} \leq\left(\underline{b}^{T} \boldsymbol{P} \underline{b}+R\right)^{-1}
$$

where

$$
\begin{aligned}
\boldsymbol{J}_{0}= & \underline{b}\left(1-\gamma_{0}^{2}\right)\left(\left(\underline{b}^{T} \boldsymbol{P} \underline{b}+R\right)\left(1-\gamma_{0}^{2}\right)\right. \\
& \left.+R_{0}^{-1} \gamma_{0}^{2}\right)^{-1} \underline{b}^{T},
\end{aligned}
$$

( $\gamma_{0}$ will be defined in due course);

3. if the inequality (22) is satisfied for $R_{0}$ and $\boldsymbol{P}$, increase $R_{0}$ and proceed to Step 2; otherwise, decrease $R_{0}$ and proceed to Step 2, checking if Step 4 is satisfied;

4. if the inequality (22) is satisfied for $R_{0}$ and $\boldsymbol{P}$, the stabilizing matrix $\boldsymbol{P}$ satisfies Eqn. (23), and there is no positive-definite solution for the pair $R_{0}$ and $\boldsymbol{P}$ for arbitrary $R_{0}^{\prime}$, where

$$
R_{0} \leq R_{0}^{\prime} \leq\left(\underline{b}^{T} \boldsymbol{P}^{*} \underline{b}+R\right)^{-1}
$$

then stop the algorithm; in this case, the state-feedback vector is given as

$$
\begin{aligned}
\underline{k}^{T}= & -\gamma^{-1}\left(1-\left(X^{-1}-R_{0}\right)\left(\left(1-\gamma_{0}^{2}\right)\right.\right. \\
& \left.\left.+\gamma_{0}^{2} R_{0}^{-1} X^{-1}\right)^{-1} \gamma_{0}^{2} R_{0}^{-1}\right) X^{-1} \underline{b}^{T} \boldsymbol{P} \boldsymbol{A},
\end{aligned}
$$

where $X=\underline{b}^{T} \boldsymbol{P} \underline{b}+R$.

The following notation has been adopted in the above algorithm (Yang et al., 2000a):

$$
\begin{aligned}
\gamma & =\frac{\varrho_{+}+\varrho_{-}}{2}, \\
\gamma_{0} & =\frac{\varrho_{+}-\varrho_{-}}{\varrho_{+}+\varrho_{-}} .
\end{aligned}
$$

The matrix $\boldsymbol{P}$ satisfying the equation

$$
\begin{aligned}
\boldsymbol{P}= & \boldsymbol{Q} \boldsymbol{A}^{T} \boldsymbol{P} \boldsymbol{A} \\
& -\boldsymbol{A}^{T} \boldsymbol{P} \underline{b}\left(\underline{b}^{T} \boldsymbol{P} \underline{b}+R\right)^{-1} \underline{b}^{T} \boldsymbol{P} \boldsymbol{A}
\end{aligned}
$$

is called the stabilizing Ricatti solution to the Ricatti equation, and all eigenvalues of the matrix $\boldsymbol{A}-\underline{b}\left(\underline{b}^{T} \boldsymbol{P} \underline{b}+\right.$ $R)^{-1} \underline{b}^{T} \boldsymbol{P} \boldsymbol{A}$ are inside the unit circle.

In addition, we have (Yang et al., 2000b)

$$
\begin{aligned}
\varrho & =\left(1+\varrho_{0}\right) \gamma, \\
\left|\varrho_{0}\right| & \leq \gamma_{0} \leq 1,
\end{aligned}
$$

where

$$
\varrho_{0}=\frac{\varrho-\gamma}{\gamma} .
$$




\section{Actuator failure vs. active constraints and undermodeling errors}

When the input signal of the actuator saturates, in amplitude-constrained control, it may be treated as a special case of actuator failure. In such a situation, it is assumed that $\gamma=\alpha$ and the constrained control signal becomes (Horla and Królikowski, 2011)

$$
u_{t}^{F}=\operatorname{sat}\left(\underline{k}^{T} \underline{x}_{t} ; \alpha\right)
$$

where sat is a function that imposes constraints on the control signal in the span of $\pm \alpha$.

A single actuator failure on the basis of (11) and (32) is presented in Fig. 3. The assumed failure model can be put in the form

$$
u_{t}^{F}=\operatorname{sat}\left(\varrho v_{t} ; \alpha\right)
$$

as a compilation of the models (32) and (11) (the dashed area in Fig. 3).

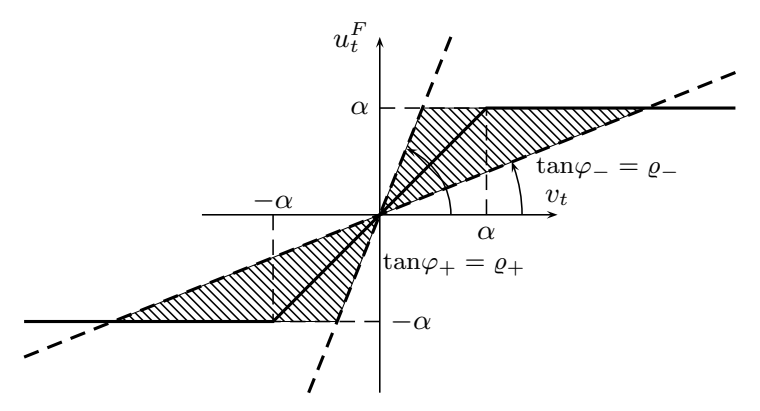

Fig. 3. Actuator failure and amplitude constraints.

The dashed cones in Fig. 3 might also depict the uncertainty introduced by using a linearized model instead of a true nonlinear model of the plant, e.g., due to the existence of the nonlinear static characteristic. Whenever control signals computed for the linearized case fail to result in expected changes in output signals due to undermodeling, one can treat this case as an actuator failure and use algorithms cited in this paper.

Figure 4 presents robustness areas for different values of $\varrho_{+}, \varrho_{-}$, making it possible to visualize the "robustness area" of the control law applied and its analogy to the actuator failure case in the controller. As has been already mentioned, the more different the values of $\varrho_{+}$and $\varrho_{-}$, the larger the cones.

\section{Experimental comparison of control strategies}

8.1. Experiment conditions. Figure 5 presents the robot during the experiment. As can be seen, there is a

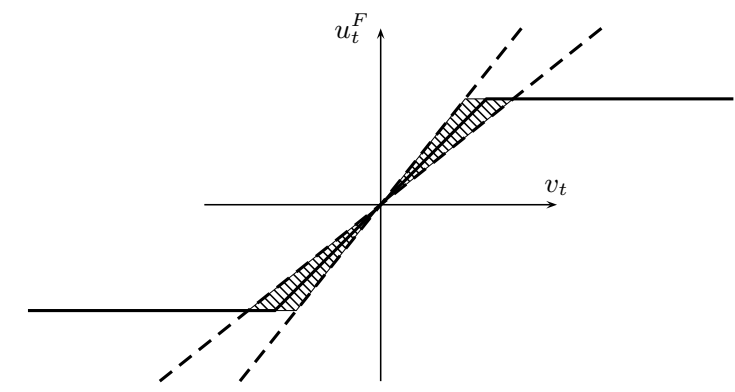

(a)

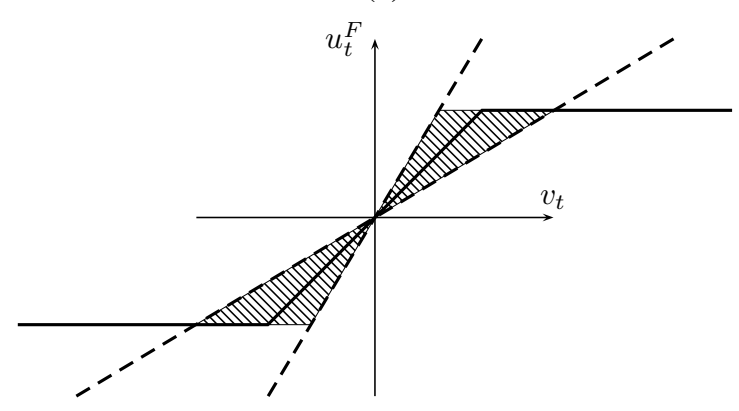

(b)

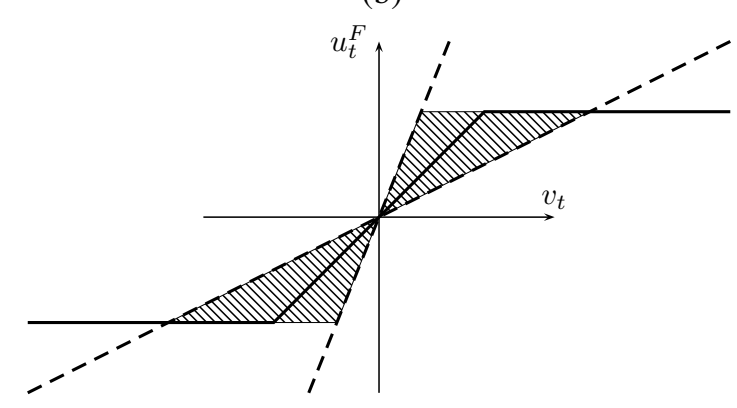

(c)

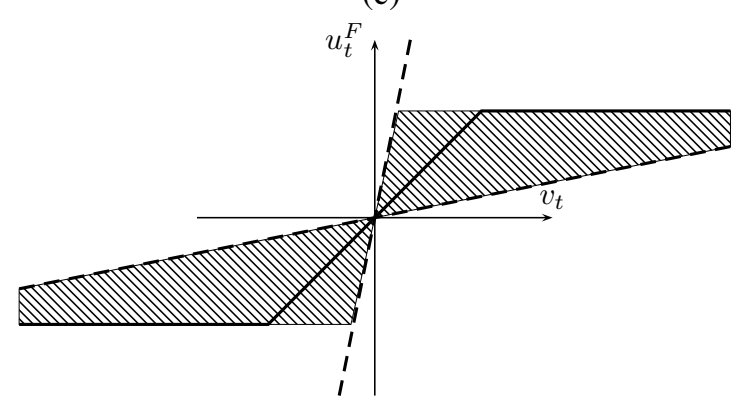

(d)

Fig. 4. Robust action areas for $\rho_{+}=1+\delta, \rho_{-}=1-\delta$ with $\delta=0.2(\mathrm{a}), \delta=0.4$ (b), $\delta=0.6$ (c),$\delta=0.8$ (d).

small block on the left which supports the construction every time right before the trigger. When the trigger appears, it immediately turns on the control system and the data acquisition one. For the next few seconds, the machine restores its upright position and is trying to reach the equilibrium point. All important signal values are 


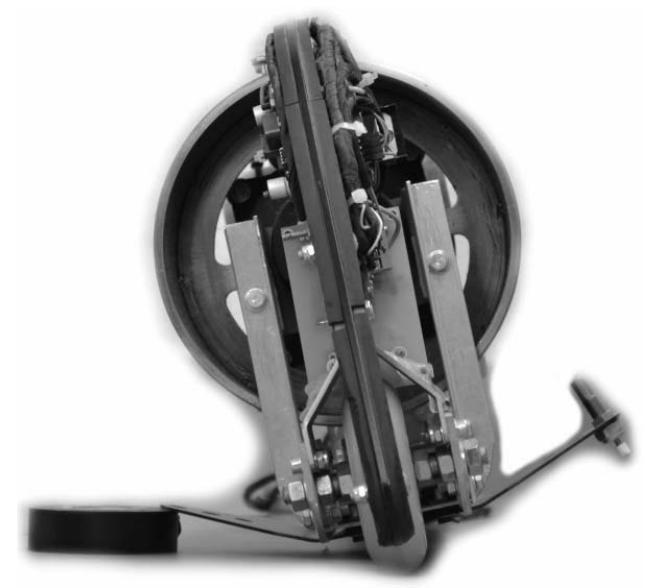

Fig. 5. Real robot during the experiment.

stored in the SD card with a frequency of $200 \mathrm{~Hz}$. The initial deflection angle in every experiment is the same $\left(\underline{x}_{1,0}=-0.06 \mathrm{rad}\right)$.

The controllers considered work in discrete-time with their output fed to zero-order hold and based on the discretised model of the linearized plant (5), (6).

During the experiment, the following parameters have been fixed: $T_{S}=0.02 \mathrm{~s}, \boldsymbol{Q}=$ $\operatorname{diag}\{1,1,0.01,0.4\}, R=10,0 \leq \delta \leq 0.8, \rho_{+}=1+\delta$, $\rho_{-}=1-\delta, \alpha=2.1 \mathrm{~A}, R_{0}=0.1, \underline{k}_{I}=[0,0,0.2,0]^{T}$, $\underline{x}_{0}=\left[-0.06 \mathrm{rad}, 0 \mathrm{rad} \cdot \mathrm{s}^{-1}, 0 \mathrm{rad}, 0 \mathrm{rad} \cdot \mathrm{s}^{-1}\right]^{T}$. For each of 18 values of $\delta$, a series of 5 experiments was carried out for both LQR and LQI controllers, producing two sets of 90 experiments. The results presented in subsequent sections are mean values from every experiment series.

It is to be stressed that $Q$ and $R$ have been chosen to assure that optimal $\underline{k}$ computed via a standard LQR procedure provides acceptable dynamics of the closed-loop system, and guarantees that the control signal does not saturate frequently and the mechanics of the machinery are far from resonant-line behavior.

8.2. Performance indices. In order to verify the behavior of the control system, the following two performance indexes were introduced:

$$
\begin{aligned}
J_{x_{i}} & =\sum_{t=0}^{N} x_{i, t}^{2} \quad(i=1, \ldots, 4), \\
J_{u} & =\sum_{t=0}^{N} u_{t}^{2},
\end{aligned}
$$

where $N$ denotes the number of samples collected at each multiplicity of $T_{S}$ from the sampled-data control system. The experiment time was equal in all cases and set to $7.5 \mathrm{~s}$. The first index is related to the energy carried by $x_{i, t}$. In the case of $x_{1}$, which has to be stabilized at zero (upright position, unstable equilibrium point); $x_{2}$ being the angular velocity of the robot, $x_{3}$ meaning the rotation angle of the reaction wheel (partially related to energy consumption in a steady-state) that also represents the gyroscopic drift and its velocity $x_{4}$, small values of the corresponding indices represent a smooth response to initial conditions.

8.3. Results. In Fig. 6, a series of plots is presented, derived from a set of $90 \mathrm{LQR}$ experiments, whereas in Fig. (7) the results for $90 \mathrm{LQI}$ experiments are depicted. As can be seen, the implemented controllers allow stabilization of the nonlinear plant, i.e., an unmanned bicycle robot, in an unstable equilibrium point. It must be noticed that for the LQR controller the mean value of the control signal after $t>2 \mathrm{~s}$ is nonzero, which causes both energy consumption and forces the reaction wheel to rotate slowly to compensate the gyroscopic drift seen in the plot of $x_{3}$. The larger the value of $\delta$, the greater the uncertainty information passed to the control system, which causes the control signal to change rapidly (there is a 25 times increase in the mean variance of a set of 5 experiments between $\delta=0$ and $\delta=0.8$, both for $t>2 \mathrm{~s}$ ), and corresponds to the action of the controller to conform to the upper boundary $(20)$. On the contrary, in the case of the LQI controller, this increase is 4 times only.

For the LQI controller, by integrating $x_{3}$ and using this information in the control law, it is assured that the mean value of the control signal in the time horizon as above is virtually zero, which results, for longer observation, in no angular change in the rotation of the reaction wheel. For a shorter period of time, swaying between the integral of $x_{3}$ and $x_{1}$ visible. Nevertheless, especially in the case of the gyroscopic drift or its initial offset, states $x_{1}$ and $x_{2}$ drift away from zero in longer time horizons in the LQR case (see Fig. 8), causing $x_{3}$ to increase also; this is not the case for the LQI strategy, where no drift in $x_{3}$ can be observed.

Mean performance indices for LQR and LQI controllers are presented in Fig. 9 The initial values, i.e. for $\delta=0$, related to plain LQR or LQI, are given in Table 1 and reflect the assumption that the linearized model (7), (8) is true. In comparison of these values with plots depicted in Fig. 9 it becomes obvious that by incorporating robustness against actuator failure, which can be treated as a discrepancy between the expected behavior of the robot with respect to its linearized model and its true response to the control signal, the performance indices are decreased in a certain span of $\delta$. It is noticeable that for approximately. $\delta=0.1$ the decrease in $J_{x_{1}}$ is nearly $10 \%$ of the value for plain LQR, whereas in the case of energy consumption it is reduced by over $30 \%$. The same phenomenon has been observed in simulations of the robot model in a sampled-data control system with the same sampling period, as reported by Horla and 

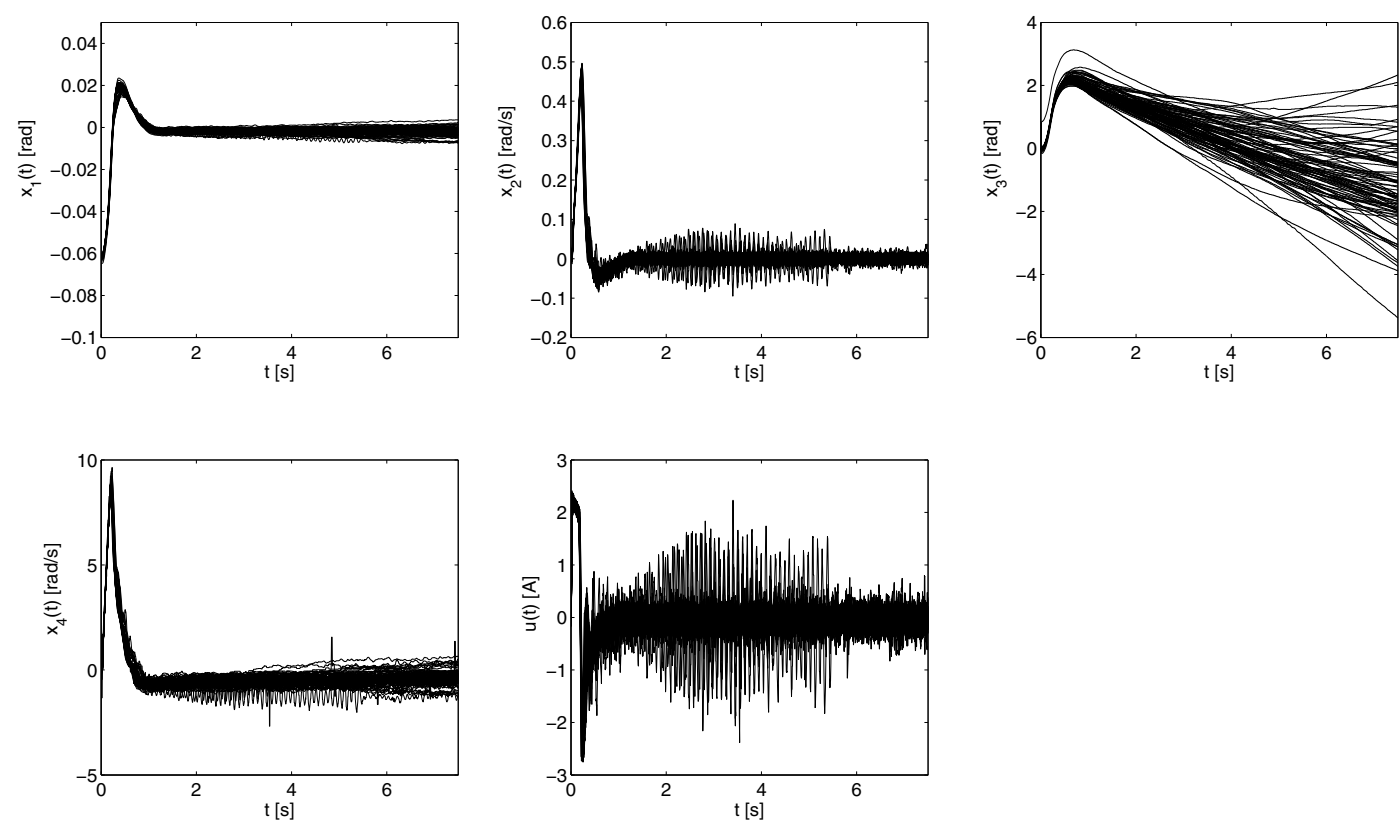

Fig. 6. Data set derived from a set of $90 \mathrm{LQR}$ experiments.
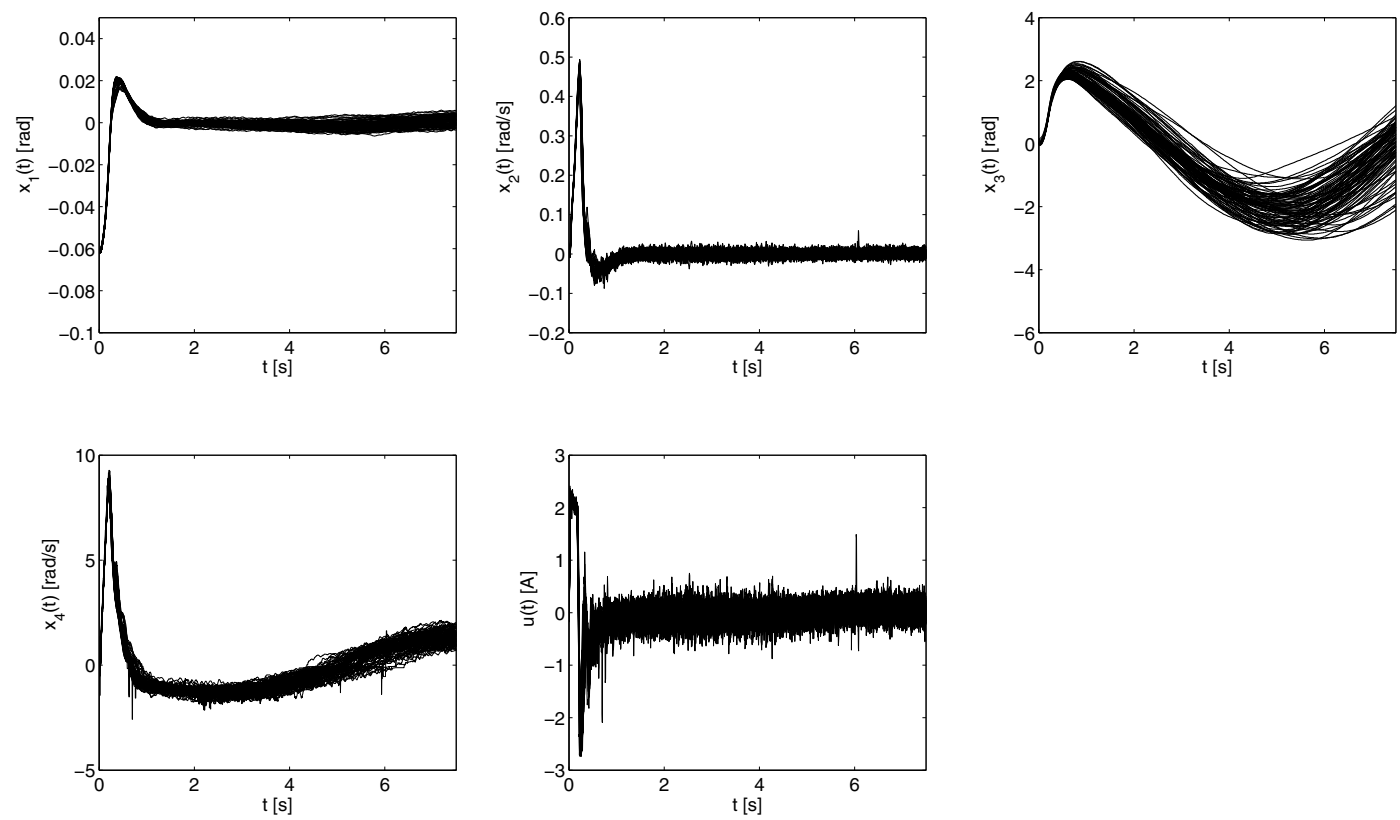

Fig. 7. Data set derived from a set of $90 \mathrm{LQI}$ experiments. 

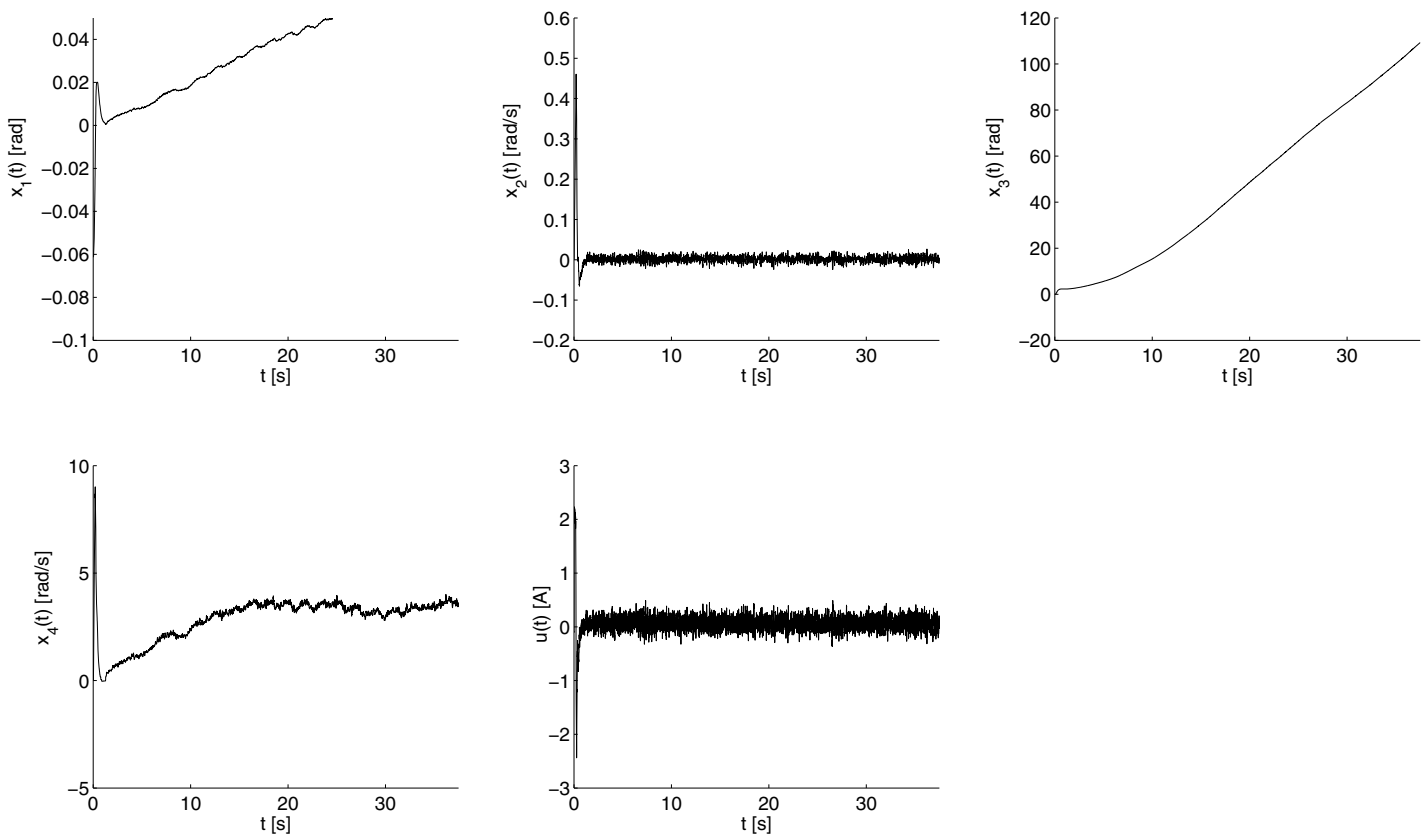

Fig. 8. Gyroscopic drift in the case of its initial offset and LQR control.
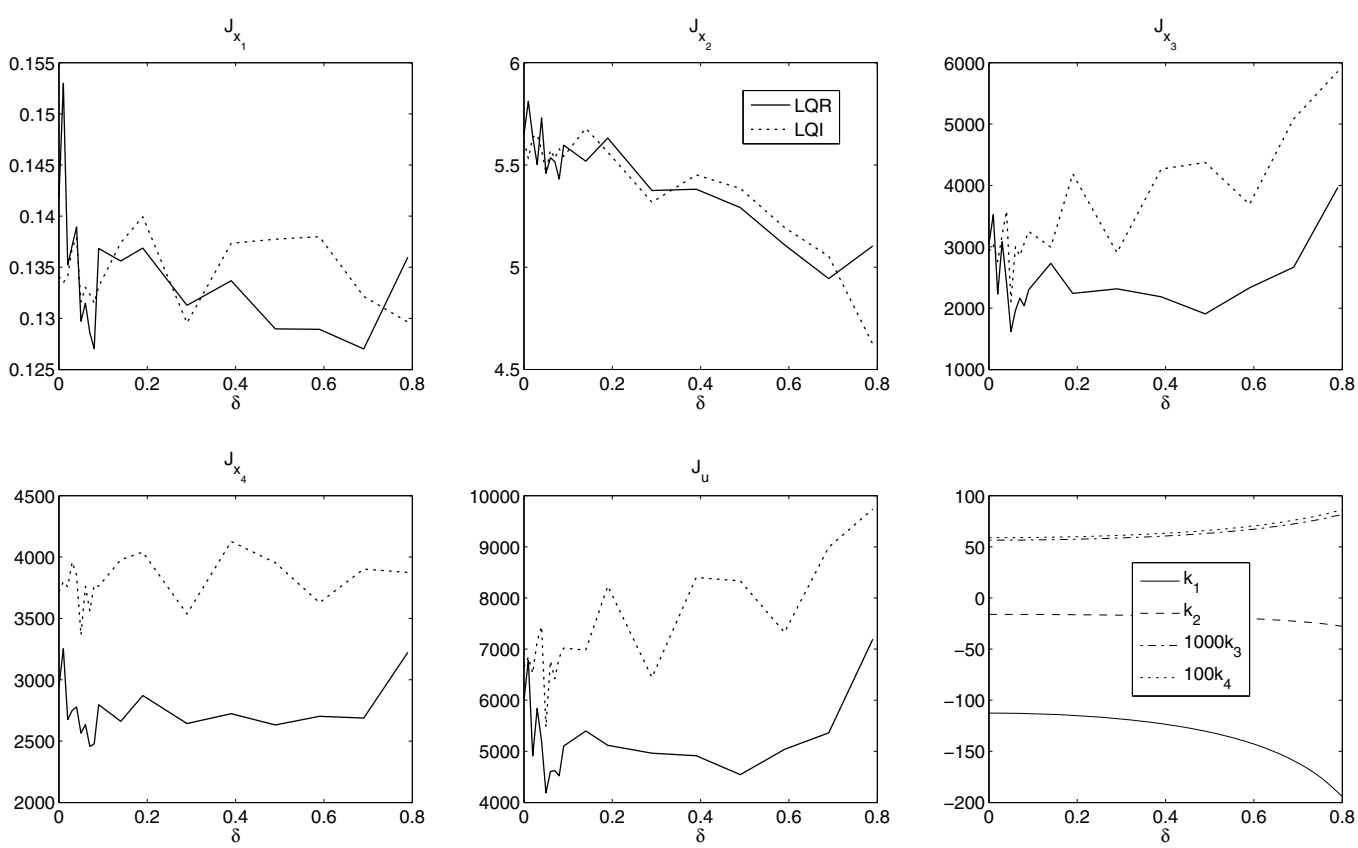

Fig. 9. Mean performance indices for the control laws considered (units have been omitted). 
Owczarkowski (2015), with a similar increase in other performance indices in the case of LQI with respect to LQR control.

From the plot of $J_{u}$ it can be observed that there is a range of values of $\delta$ for which the performance index is small with respect to its value for $\delta=0$, whereas for large values of $\delta$ it increases and causes abrupt changes in signals as reported above, resulting in an increase in other performance indices. This is related to the inability of the control law to encompass such a degree of uncertainty which is 'fed' to the control law. The rapid changes in signals for large $\delta \mathrm{s}$ are a result of an increase in components of $\underline{k}$, as in the last plot in Fig. 9 .

Table 1. Values of performance indices for $\delta=0$.

\begin{tabular}{|c||c|c|c|c|c|}
\hline control law & $J_{x_{1}}$ & $J_{x_{2}}$ & $J_{x_{3}}$ & $J_{x_{4}}$ & $J_{u}$ \\
\hline \hline LQR & 0.1418 & 5.4680 & 3047 & 2944 & 5998 \\
LQI & 0.1341 & 5.6187 & 2926 & 3716 & 6648 \\
\hline
\end{tabular}

\section{Conclusions}

By introduction of an actuator failure model into LQR and LQI control laws it is possible to reduce performance indices, especially energy consumption. This reduction is mainly due to using a linearized robot model in a sampled-data controller and by using the notion of the actuator failure. Since it might be treated as a means to mimic modeling imperfections, as well as the presence of nonlinearities in the control system, by increasing the robustness of the control system we obtain slower transients and the bicycle robot stabilizes at an unstable equilibrium point. In addition, by introducing an integral term to LQR control, the gyroscopic drift is eliminated, causing again smaller energy consumption in a longer time horizon of the experiment.

In further research, it should be verified if a similar approach enables us to improve LQR controller results in the case of a 4DOF robot, enabling us to modify the steering wheel angular change, as well as the linear speed of the robot.

The presented approach is computationally simple and requires only necessary calculations of $\underline{k}$ off-line and storing them for future use, which makes it applicable to real-time control systems.

\section{References}

Block, D.J., Åström, K.I. and Spong, M.W. (2007). The reaction wheel pendulum, Synthesis Lectures on Controls and Mechatronics 1(1): 1-105, DOI: 10.2200/S00085ED1V01Y200702CRM001.

Chang, S. and Peng, T. (1972). Adaptive guaranteed cost control of systems with uncertain parameters, IEEE Transactions on Automatic Control 17(4): 474-483, DOI: 10.1109/TAC.1972.1100037.
Drapikowski, P., Goślinski, J. and Owczarkowski, A. (2012). Control and model parameters identification of inertia wheel pendulum, Proceedings of the 9th International Conference on Informatics in Control (ICINCO), Rome, Italy, pp. 574-579.

Horla, D. and Królikowski, A. (2011). Discrete-time LQG control with actuator failure, Proceedings of the 8th International Conference on Informatics in Control, Automation and Robotics, Noordwijkerhout, The Netherlands, (on CD-ROM).

Horla, D. and Owczarkowski, A. (2015). Robust LQR with actuator failure control strategies for $4 \mathrm{DoF}$ model of unmanned bicycle robot stabilised by inertial wheel, 2015 International Conference on Industrial Engineering and Systems Management (IESM), Seville, Spain, pp. 998-1003, DOI: 10.1109/IESM.2015.7380276.

Kwakernaak, H. and Sivan, R. (1972). Linear Optimal Control Systems, Wiley-Interscience, Hoboken, NJ.

Owczarkowski, A., Lis, M. and Kozierski, P. (2014). Tracking control of an inertial wheel pendulum by LQR regulation, Proceedings of the 19th International Conference on Methods and Models in Automation and Robotics, Międzyzdroje, Poland, (on CD-ROM).

Petersen, I. and McFarlane, D. (1992). Optimizing the guaranteed cost in the control of uncertain systems, in $\mathrm{M}$. Mansour et al. (Eds.), Robustness of Dynamical Systems with Parameter Uncertainties, Brikhäuser, Boston, MA, pp. 241-250.

Smerpitak, K., Ukakimparn, P., Trisuwananwat, T. and Trakoonkootaworn, S. (2012). An unmanned bicycle versus linear quadratic optimal controls, Proceedings of the 12th International Conference on Control, Automation and Systems, JeJu Island, Korea, pp. 1337-1341.

Xie, L. and Soh, Y. (1995). Guaranteed cost control of uncertain discrete-time systems, Control Theory and Advanced Technology 10(4): 1235-1251.

Yang, J., Lee, S., Kim, S., Lee, Y. and Kwon, O. (2011) Linear controller design for circular motion of unmanned bicycle, Proceedings of the 11th International Conference on Control, Automation and Systems, Gyeonggi-do, Korea, pp. 893-897.

Yang, Y., Wang, J. and Soh, Y. (2000a). Reliable LQG control with sensor failures, IEE Proceedings: Control Theory and Applications 147(4): 433-439.

Yang, Y., Yang, G. and Soh, Y. (2000b). Reliable control of discrete-time systems with actuator failures, IEE Proceedings: Control Theory and Applications 147(4): 428-432.

Zuo, Z., Ho, D. and Wang, Y. (2010). Fault tolerant control for singular systems with actuator saturation and nonlinear perturbation, Automatica 46(3): 569-576. 


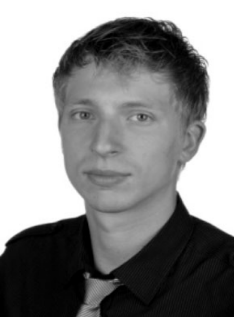

Adam Owczarkowski is finishing his $\mathrm{Ph} . \mathrm{D}$. studies at Poznań University of Technology, Faculty of Electrical Engineering, Institute of Control and Information Engineering. He holds an M.Sc. degree in control from the Poznań University of Technology. He focuses his work on control strategies for underactuated robots and is also interested in linear quadratic control, non-linear control, object modeling, embedded system programming and machine intelligence.

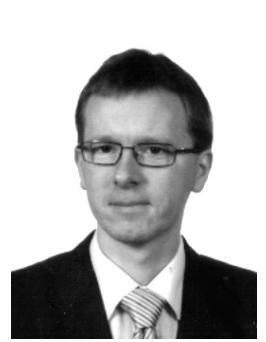

Dariusz Horla is with the Institute of Control and Information Engineering of the Poznań University of Technology. He received his M.Sc. in 2002, his Ph.D. in 2005 and his D.Sc. in 2013, all from the Faculty of Electrical Engineering of the Poznan University of Technology. His research interests include anti-windup compensation, optimal control, linear matrix inequalities, optimization theory, and adaptive control.

Received: 30 March 2015

Revised: 8 July 2015

Accepted: 9 October 2015 